OPEN ACCESS

Edited by:

Jose Ruben Morones-Ramirez,

Autonomous University of Nuevo

León, Mexico

Reviewed by:

Run Zhang,

The University of

Queensland, Australia

Wei He,

China Pharmaceutica

University, China

*Correspondence:

Claudio Cabral-Romero claudio.cabralrm@uanl.edu.mx

Specialty section: This article was submitted to Biomedical Nanotechnology,

a section of the journal

Frontiers in Nanotechnology

Received: 25 November 2020

Accepted: 16 March 2021

Published: 12 April 2021

Citation:

Cabral-Romero C, García-Cuellar CM,

Hernandez-Delgadillo $R$,

Sánchez-Pérez Y, Meester I,

Solís-Soto JM, Pineda-Aguilar N and

Chellam S (2021) Synergistic

Antitumor Activity of

Gramicidin/Lipophilic Bismuth Nanoparticles (BisBAL NPS) on

Human Cervical Tumor Cells.

Front. Nanotechnol. 3:633604.

doi: 10.3389/fnano.2021.633604

\title{
Synergistic Antitumor Activity of Gramicidin/Lipophilic Bismuth Nanoparticles (BisBAL NPs) on Human Cervical Tumor Cells
}

\author{
Claudio Cabral-Romero ${ }^{1 *}$, Claudia María García-Cuellar ${ }^{2}$, Rene Hernandez-Delgadillo ${ }^{1}$, \\ Yesennia Sánchez-Pérez ${ }^{2}$, Irene Meester ${ }^{3}$, Juan Manuel Solís-Soto ${ }^{1}$, \\ Nayely Pineda-Aguilar ${ }^{4}$ and Shankararaman Chellam ${ }^{5}$
}

' Laboratorio de Biología Molecular, Facultad de Odontología, Universidad Autónoma de Nuevo León, UANL, Monterrey, Mexico, ${ }^{2}$ Subdirección de Investigación Básica, Instituto Nacional de Cancerología, Ciudad de Mexico, Mexico, ${ }^{3}$ Departamento de Ciencias Básicas, Universidad de Monterrey, San Pedro Garza García, Mexico, ${ }^{4}$ Centro de Investigación en Materiales Avanzados, S.C. (CIMAV), Unidad Monterrey, Monterrey, Mexico, ${ }^{5}$ Texas A\&M University, College Station, TX, United States

The objective of this study was to study the synergistic antitumor effect of lipophilic bismuth nanoparticles (BisBAL NPs) with the antibiotic solution Neo-Poly gramicidin on human cervical tumor cells. The effect of BisBAL NPs and Neo-Poly gramicidin solution on cervical cancer cell line (HeLa) was determined by the MTT cell viability assay and fluorescence microscopy. After a 24-h exposure to $0.1 \times$ Neo-Poly gramicidin HeLa cell growth decreased 94\%. Fluorescence microscopy confirmed the antitumor effect cell death was higher among treated than among non-treated cells cells. Individually, gramicidin $(0.04 \mathrm{mg} / \mathrm{mL})$ inhibited HeLa tumor cell growth most $(40 \%)$, and neomycin $(0.04 \mathrm{mg} / \mathrm{mL})$ least $(21 \%)$. Gramicidin $(0.3 \mathrm{mg} / \mathrm{mL})$ in combination with different concentrations $(1-150 \mu \mathrm{M})$ of BisBAL NPs had a synergistic antitumor effect against HeLa cells, reaching an $<86 \%$ tumor growth inhibition. As far as we know, we are the first to describe the antitumor activity of the antibiotic Neo-Poly gramicidin on a human cervical cancer cell line. The action mechanism of gramicidin/BisBAL NP is based on a strong damage on cell membrane and nucleus of tumor cells. A synergistic effect of gramicidin with BisBAL NPs may be useful as an alternative therapy for cervical cancer patients.

Keywords: antitumor activity, bismuth nanoparticles, cervical cancer, neomycin, polymyxin B, gramicidin, synergistic effect

\section{INTRODUCTION}

Cervical cancer is the fourth most prevalent cancer among females and is the second cause of cancer death in developing countries (Torre et al., 2015). In 2013, 485,000 patients were diagnosed with cervical cancer in 2013 and 236,000 died worldwide. Cervical cancer is strongly associated with human papillomavirus infection, which causes benign or malign lesions: Examples of the latter are vaginal and vulvar cancers, which are caused by human papilloma virus subtypes 16 and 18 (Clarke et al., 2012). 
According to the International Federation of Gynecology and Obstetrics guidelines, cervical cancer patients can be classified in: early-stage disease, locally advanced disease and advanced disease (Serrano-Olvera et al., 2015). Thirteen percent of cervical cancer patients are diagnosed at advanced stages. Many of them will develop recurrent disease at early stage of treatment and $~ 90 \%$ of these females will die within 5 years (Elit et al., 2009; Adegoke et al., 2012). Only $16.5 \%$ of metastatic cervical patients will survive in comparison with $91.5 \%$ for localized cervical cancer (Ferlay et al., 2013).

Treatment options for cervical cancer include surgery, radiotherapy, and chemotherapy. Although surgery by local ablation is one of most common treatments for cervical neoplasia (Martin-Hirsch et al., 2013), not all patients are candidates to surgery, because of age, tumor size, stage of disease, etc. The employment of radiotherapy must consider the tumor characteristics and possible damage to adjacent non-tumor cells. Chemotherapy against cervical cancer is an important treatment option, but systemic therapy has several limitations, such as a lack of target specificity, the development of resistance, and severe side effects (Nascimento et al., 2017). The anti-cancer drug, docetaxel causes liver injury (Wang et al., 2012; Yared and Tkaczuk, 2012), which make patients abandon treatment. The advantages of its topical alternative include reaching locally higher concentrations with less adverse effects to non-exposed normal cells. However, so far there is no topical antitumor drug for cervical cancer.

The Topical chemotherapy has been successfully applied to ophthalmic infections (Bosscha et al., 2004). A combination of the antibiotics neomycin, polymyxin $\mathrm{B}$, and gramicidin (Neo-Poly gramicidin) had broad spectrum antibacterial activity (Gibson, 1983). There are no reports about possible antitumor activity of Neo-Poly gramicidin. Doxorubicin is the best example of an antibiotic that also has antitumor activity (Rivankar, 2014). Unfortunately, the excessive toxicity of doxorubicin continues being a challenge in clinical practice (Waterhouse et al., 2001). Previously, we reported the selective antitumor effect of bismuth lipophilic nanoparticles (BisBAL NPs) on human breast and cervical cancer cells as well as other types of human cancer (Hernandez-Delgadillo et al., 2018; Cabral-Romero et al., 2020). However, the antitumor effect of BisBAL NPs in combination with other drugs against cervical cancer cells has not been explored. The aim of this study was to verify the possible synergistic antitumor activity of gramicidin in combination with BisBAL NPs on human cervical cancer cells.

\section{MATERIALS AND METHODS \\ Neo-Poly Gramicidin Solution}

A stock solution (SULNED, Pizzard Lab., Jalisco, México) containing $175 \mathrm{mg} / \mathrm{mL}$ neomycin, $5,000 \mathrm{U} / \mathrm{mL}$ polymyxin $\mathrm{B}$, and $25 \mathrm{mg} / \mathrm{mL}$ gramicidin (all from Sigma Aldrich, MO, USA). was diluted serially in sterile distilled water before each experiment.

\section{Synthesis and Characterization of BisBAL NPs}

BisBAL NPs were synthesized and characterized as previously was described at earlier reports (Badireddy et al., 2014; CabralRomero et al., 2020). The BisBAL NP's morphology was analyzed with scanning electron microscopy (SEM; FEI Tecnai G2 Twin, Hillsboro, OR, USA; $160 \mathrm{kV}$ accelerating voltage). Energydispersive X-ray spectroscopy (EDS) SEM (EDS; Oxford INCA $\mathrm{X}$-Sight, Tubney Woods, UK) was used for the elemental analysis of samples. X-ray diffractometry patterns were obtained from water-washed (three centrifugation cycles, $16,100 \times g, 30 \mathrm{~s}$ ), airdried BisBAL NPs (deposited several times on a glass slide overnight) using an X-ray diffractometer (Panalytical X'Pert PRO MRD) equipped with $\mathrm{Cu} \mathrm{K} \alpha$ as an $\mathrm{X}$-ray source $(\lambda=1.541874 \AA)$.

\section{Cell Culture}

The cervical cancer cell line (HeLa) used in this study was purchase from the American Type Culture Collection (ATCC; CCL-2; Rockville, MD, USA). The HeLa cells were cultured in Dulbecco's modified Eagle's medium/Ham's F12 (DMEM/F12) supplemented with $10 \%$ fetal bovine serum (FBS; GibcoInvitrogen, Carlsbad, CA, USA), $100 \mathrm{U} / \mathrm{mL}$ penicillin, $100 \mu \mathrm{g} / \mathrm{mL}$ streptomycin, and $0.25 \mu \mathrm{g} / \mathrm{mL}$ amphotericin B (Sigma-Aldrich Corporation, St. Louis, MO, USA) in cell culture flasks (Corning Inc., Corning, NY, USA) at $37^{\circ} \mathrm{C}$ in a humidified atmosphere with $5 \% \mathrm{CO}_{2}$ for 2 days until get a confluent monolayer.

\section{MTT Cell Viability Assay}

The antitumor activity of Neo-Poly gramicidin (in solution with the three antibiotics or individually) on HeLa cells employing the cell viability 3-(4,5-dimethylthiazol-2-yl)-2,5diphenyltetrazolium bromide (MTT) assay (Biotium, Hayward, CA, USA) (Liu et al., 1997; Hernandez-Delgadillo et al., 2018). The experimental groups were as follow: Neo-Poly gramicidin solution with serial dilutions $\left(1: 10-1: 10^{8}\right), 12 \mu \mathrm{M}$ of docetaxel (Zurich Pharma, Mexico city, Mexico) as positive control, drugfree as growing control and $0.3 \mathrm{mg} / \mathrm{mL}$ gramicidin + BisBAL NPs $(1,10,25,50,100$, and $150 \mu \mathrm{M})$. We added $1 \times 10^{5} \mathrm{HeLa}$ cells in $100 \mu \mathrm{L}$ of culture medium to each well of a 96-well plate, and incubated at $37^{\circ} \mathrm{C}, 5 \% \mathrm{CO}_{2}$ for $24 \mathrm{~h}$. Next, cell viability was analyzed by MTT assay according to the provider's instructions. To quantify the reduced MTT, the absorbance at $570 \mathrm{~nm}\left(\mathrm{~A}_{570}\right)$ was measured with a microplate absorbance reader (Biotek, Winooski, VT). The experiment was performed in triplicate and cell viability was obtained from measured absorbance and expressed as cell viability percentage.

\section{Cell Morphology by Fluorescence Microscopy}

Cell morphology of HeLa cells was analyzed after treatment with Neo-Poly gramicidin/BisBAL by using fluorescein diacetate (FDA) or intracellular calcein-AM assay (Rene et al., 2016) and fluorescence microscopy. The experimental groups were following; Neo-Poly gramicidin stock solution (1:10 and 1:10 $)$, and drug-free was employed as growing control. $1 \times 10^{5} \mathrm{HeLa}$ cells in $100 \mu \mathrm{L}$ of culture medium to each well of a 96-well 
plate were exposed to each drug for $24 \mathrm{~h}$. After incubation, cells were washed three times with PBS and stained with stained with FDA (Sigma-Aldrich Corporation, St. Louis, MO) and 4,6-diamidino-2-phenylindole (DAPI) (Abcam Inc., Cambridge, UK) for $30 \mathrm{~min}$. at $37^{\circ} \mathrm{C}$. Next, cells were washed again with PBS and air-dried in the dark. Cell morphology was observed with FITC and DAPI filters at $485 \mathrm{~nm}$ and $358 \mathrm{~nm}$, respectively (Thornwood, NY).

\section{Cell Membrane Permeability by Fluorescence Microscopy}

Calcein AM assay and fluorescence microscopy were used to study the cell membrane integrity of HeLa cells after treatment with gramicidin alone or in combination with BisBAL NPs. The experimental groups were as follow: $50 \mu \mathrm{M}$ BisBAL NP, 0.3 $\mathrm{mg} / \mathrm{mL}$ gramicidin, 1,200 $\mu \mathrm{M}$ chlorhexidine (CHX) (Ultradent Products, South Jourdan, UT) as positive control and drug-free as growing control. We added $1 \times 10^{5} \mathrm{HeLa}$ cells in $100 \mu \mathrm{L}$ of culture medium to each well of a 96-well plate and incubated at $37^{\circ} \mathrm{C}, 5 \% \mathrm{CO}_{2}$ for $24 \mathrm{~h}$. After incubation, cells were washed three times with PBS and stained with $2 \mu \mathrm{M}$ Calcein AM (Biotium, Hayward, CA, USA) for $30 \mathrm{~min}$. at $37^{\circ} \mathrm{C}$ (Bozyczko-Coyne et al., 1993). Nuclei of tumor cells were stained with $1 \mu \mathrm{g} / \mathrm{mL}$ 4',6-diamidino-2-phenylindole (DAPI) (Abcam Inc., Cambridge, UK) (Kubista et al., 1987). Next, cells were washed again with PBS and air-dried in the dark. Cell morphology was observed with FITC and DAPI filters at 485 and $358 \mathrm{~nm}$, respectively (Thornwood, NY).

\section{Genotoxicity by Comet Assay and Fluorescence Microscopy}

The possible damage on genomic DNA of HeLa cells treated with gramicidin alone or mixed with BisBAL NPs was determined by comet assay and fluorescence microscopy. The experimental groups were following; $100 \mu \mathrm{M}$ BisBAL NP, 0.3 $\mathrm{mg} / \mathrm{mL}$ gramicidin, Mix BisBAL NP/gramicidin, 1,200 $\mu \mathrm{M}$ Chlorhexidine (CHX; Ultradent Products, South Jourdan, UT) as a positive control and drug-free was employed as growing control. $1 \times 10^{5} \mathrm{HeLa}$ cells in $100 \mu \mathrm{L}$ of culture medium to each well of a 96-well plate were exposed to each treatment for $24 \mathrm{~h}$. After incubation, genomic DNA damage was determined with the Oxiselect ${ }^{\mathrm{TM}}$ Comet Assay Kit (Cell Biolabs, Inc., CA, USA) according to the provider's instructions (Ostling and Johanson, 1984). Cell suspensions were mixed with liquefied Comet Agarose at a 1:10 ratio (v/v), and pipetted on an OxiSelect Comet Slide $(75 \mu \mathrm{L} /$ well). After a 15 -min embedding step ( $4^{\circ} \mathrm{C}$, dark, horizontal position), cells were lysed $(25 \mathrm{~mL}$ lysis buffer/slide, 30 -min incubation, $4^{\circ} \mathrm{C}$, dark, horizontal position) and treated with an alkaline solution $\left(25 \mathrm{~mL} /\right.$ slide, $30 \mathrm{~min}, 4^{\circ} \mathrm{C}$, dark) to relax and denature the DNA. Finally, the samples are electrophoresed in a horizontal chamber $(300 \mathrm{~mA}$ for $30 \mathrm{~min}$ ) to separate intact DNA from damaged fragments. Samples were washed with sterile milliQ water, and treated with $70 \%$ cold ethanol for $15 \mathrm{~min}$, and left to dry at room temperature. Comets were stained with DAPI $(100 \mu \mathrm{L} /$ well $)$ before being viewed with an epifluorescence microscope using a DAPI filter (Thornwood, NY, USA).

\section{Statistical Analysis}

One-Way Analysis of Variance (ANOVA) with Tukey HSD test was used to compare groups. For all statistical tests, a significance level of $\alpha=0.05$ was considered.

\section{RESULTS}

\section{Characterization of BisBAL NPs}

The obtained BisBAL NPs were spherical with an average diameter of $70 \mathrm{~nm}$ (Figures 1A,B). Bismuth presence was confirmed by EDS-SEM (Figure 1C). Also bismuth identity was supported by X-ray diffractometer pattern of BisBAL NPs (Figure 1D). The BisBAL NPs formed electrodense clusters, a typical characteristic of this kind of nanostructures. A fresh batch of BisBAL NPs was used to study the antitumor activity on human cervical cancer cells.

\section{Antitumor Activity of Neo-Poly Gramicidin}

The Neo-Poly gramicidin mix had a dose dependent antitumor effect as of the dilution $1: 10^{7}$, which inhibited HeLa growth with $21 \%$ (Figure 2A). The $10^{-1}$ dilution of Neo-Poly gramicidin had the strongest effect which was similar to the positive control, $12 \mu \mathrm{M}$ docetaxel. Both inhibited HeLa cell growth with $92 \%(p$ $<0.0001$ ) (Figure 2A). Thus, the Neo-Poly gramicidin mix had a dose-dependent antitumor activity on HeLa cells and competed in efficacy with a commercial antitumor drug.

\section{Cell Morphology}

Cell morphology studies after a 24-h exposure to Neo-Poly gramicidin dilutions confirmed tumor cell death. Growth control cells had the typical morphology of epithelial cells with a welldefined nucleus, but cells exposed to a $1 \times 10^{-5}$ dilution of the Neo-Poly gramicidin stock solution were spherical with multinuclear blebs; classical cytopathic characteristics previous to cell death (Figure 2B). Exposure to a $1 \times 10^{-1}$ dilution of the Neo-Poly gramicidin solution resulted in cellular debris against a dark background (Figure 2B).

\section{Antitumor Activity of Neomycin, Polymixin B, and Gramicidin Individually}

To verify whether all or only some of the antibiotics were responsible for the antitumor activity of the Neo-Poly gramicidin mixture, we analyzed the antitumor potential individually. Neomycin inhibited HeLa cell growth least, the highest concentration of neomycin $(3 \mathrm{mg} / \mathrm{mL})$ inhibited cell number by $37 \%$ (Figure 3). Polymixin B performed better and reduced cell growth $56 \%$ reduction $(p<0.0001)$ at $0.75 \mathrm{mg} / \mathrm{mL}$ (Figure 4 ). Gramicidin inhibited HeLa cell growth best; achieving a $40 \%$ cell growth inhibition with only $0.04 \mathrm{mg} / \mathrm{mL}(p<0.0001)$ (Figure 5). Altogether, these results suggest that gramicidin is the antibiotic with highest antitumor potential against human cervical cancer cells. 


\section{Characterization of BisBAL NPs by SEM-EDX}

A



B

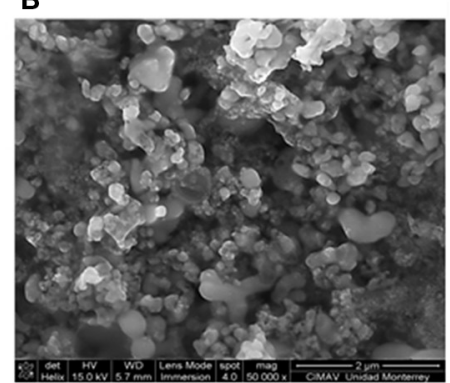

C

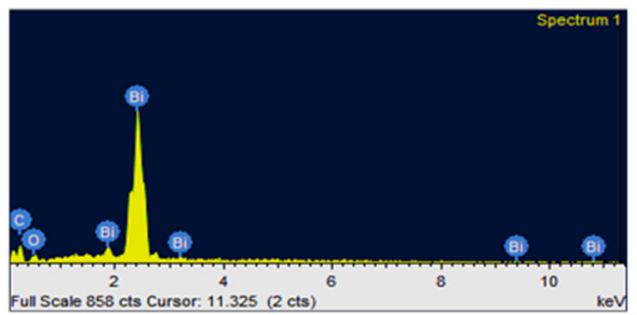

D

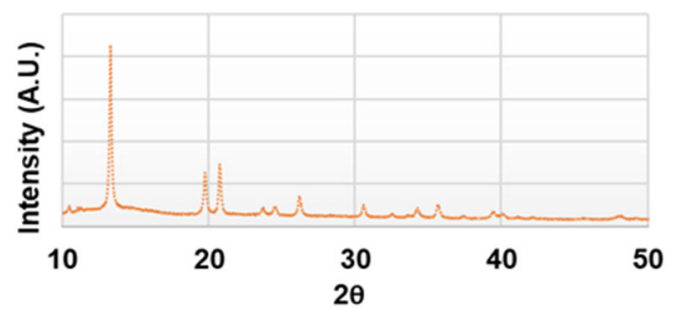

FIGURE 1 | Characterization of BisBAL NPs by scanning electron microscopy (SEM). (A,B) The shape, size, and distribution of BisBAL NPs were obtained by SEM. (C) The specific presence of bismuth was confirmed by energy-dispersive X-ray spectroscopy (SEM-EDS). (D) X-ray diffractometry pattern of BisBAL NPs.

\section{Synergistic Antitumor Activity of Gramicidin With BisBAL NPs}

Based on the above result, we verified a possible synergistic antitumor activity of gramicidin $(0.3 \mathrm{mg} / \mathrm{mL})$ in combination with different concentrations of BisBAL NPs NPs $(1-150 \mu \mathrm{M})$. As of $10 \mu \mathrm{M}$ of BisBAL NPs, tumor cell growth inhibition reached 86\% ( $p<0.0001)$ (C-index 81.6\%) (Figure 6). The antitumor potential obtained with gramicidin/BisBAL NPs was better than cancer drug docetaxel. These data suggest that the antitumor activity of gramicidin increased in combination with BisBAL NPs.

\section{Cell Membrane Permeability Assays}

To get an insight about the action mechanism of the antitumor activity of gramicidin and gramicidin/BisBAL NPs, Calcein AM assays were carried out to evaluate cell membrane permeability. Our findings showed completed destruction of cell membrane when HeLa cells were exposed to gramicidin or gramicidin/BisBAL NPs (Figure 7). Nuclei seemed unaffected (Figure 7).

\section{Genotoxic Assays}

The possible genotoxicity of gramicidin, alone and in combination with BisBAL NPs, was analyzed with the comet assay and fluorescence microscopy. A 24-h exposure to BisBAL NPs was genotoxic as can be inferred from the classical stela, also observed in the chlorhexidine positive control of genotoxicity
(Figure 8). On the other hand, a 24-h exposure to gramicidin did not cause nuclear damage (Figure 8).

\section{DISCUSSION}

Despite the availability of the HPV vaccine, cervical cancer continues being an important public health challenge worldwide with high rates of mortality in developing countries (Villa et al., 2020). Chemotherapy is followed of surgery among the different options for the treatment of cervical cancer patients, although conventional antitumor agents can be highly cytotoxic, promoting the leave of therapy (Seol et al., 2014). The inherent deficiencies of conventional chemotherapy lead to emerge nanomedicine (Shi et al., 2017). It holds the promise of develop "smart" drugs to treat several diseases including human cancer. More recent advances in biocompatibility of nanostructures employed as nanocarriers have attracted the attention to clinical therapeutics. Recently, our group described the antitumor activity of BisBAL NPs on different types of cancer including cervical cancer (Cabral-Romero et al., 2020). However, synergistic effect between bismuth nanoparticles and antibiotics has not been extensively studied. Therefore the combination of nanostructures and antibiotics could result in higher antitumor effectiveness and less non-desired effects on non-tumor cells.

In this work we report for first time the antitumor activity of the antibiotic mixture composed of neomycin, polymixin $B$ and gramicidin on a human cervical cancer cell line. We were inspired by the antitumor activity of another antibiotic, 
A 100

\section{Antitumor effect of Neo-Poly gramicidin on human cervix cancer cells}

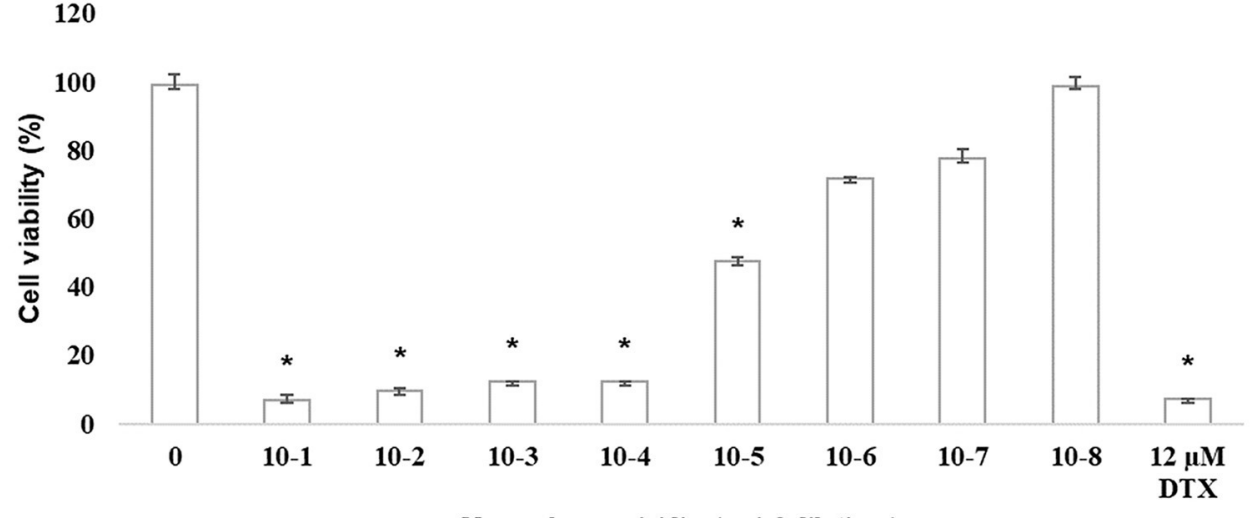

B

FDA

DAPI

Merge
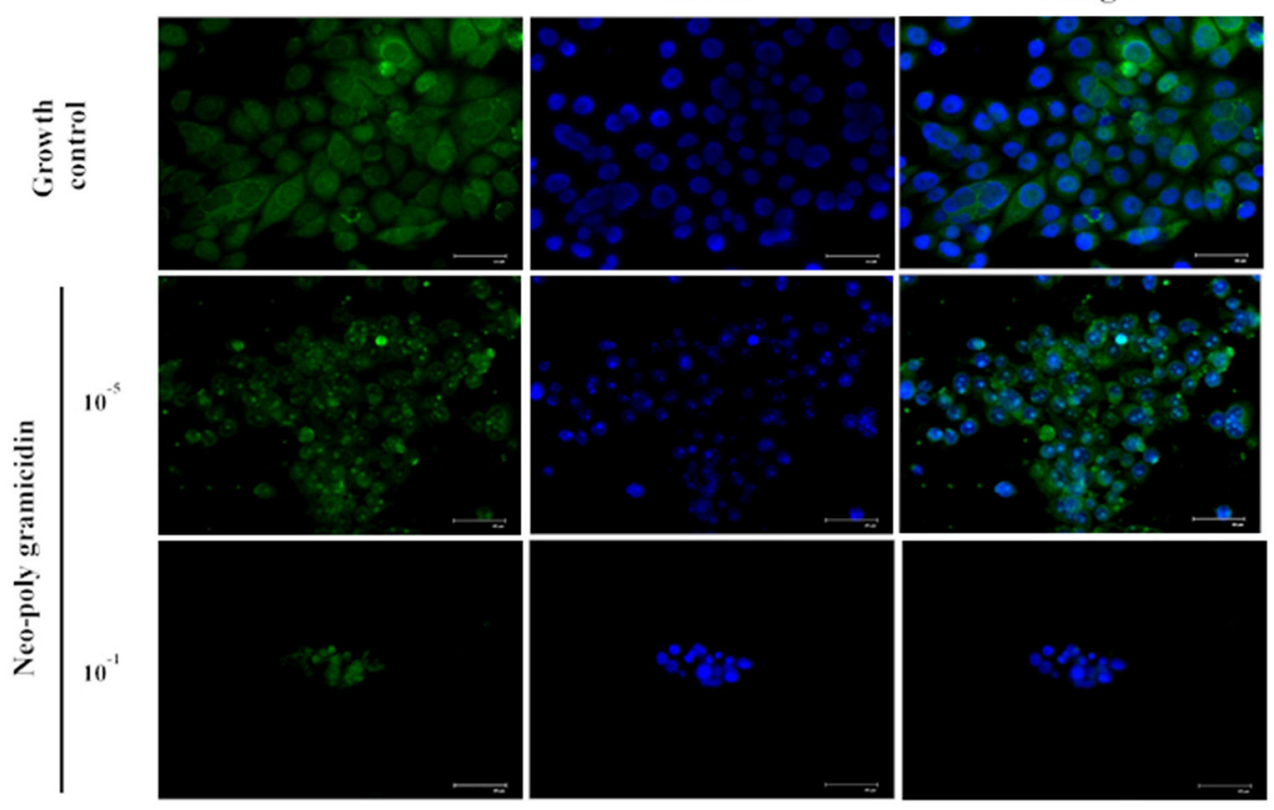

FIGURE 2 | Antitumor effect of Neo-Poly gramicidin on HeLa cell line. (A) Cell viability (via MTT assay) after a 24-h exposure to serial dilutions of Neo-Poly gramicidin $\left(10^{-1}\right.$ to $\left.10^{-8}\right)$ and $12 \mu \mathrm{M}$ docetaxel (DTX; positive control of cytotoxicity). Readings were performed in triplicate. Results are representative of three independent experiments. The antitumor effect of Neo-Poly gramicidin was dose dependent and significant as of dilution 1:105 ( $p<0.0001)$. Asterisks indicate statistical differences $(\alpha=0.05)$. (B) Cell morphology after a 24-h exposure to dilutions of Neo-Poly gramicidin $\left(10^{-1}\right.$ and $\left.10^{-5}\right)$. Untreated cells served as a growth control. nuclei are stained blue with 4,6-diamidino-2-phenylindole (DAPI). FDA, fluorescein diacetate (green). Bar $=125 \mu \mathrm{m}$.

doxorubicin or Adriamycin, which was reported in Kitaura et al. (1972). Doxorubicin is applied by injection, but we were looking for topical antibiotic application to avoid systemic adverse effects and to increase local delivery. The Neo-Poly gramicidin mixture is used topically, as eye drops, to treat ophthalmic infections. We found that a dilution of $1: 10^{4}$ of the NeoPoly gramicidin antibiotic solution inhibited $87 \%$ of the tumor growth. Previously, it was described that neomycin inhibits the angiogenic activity of fibroblasts and epidermal growth factors (Hu, 2001), supporting our hypothesis of antitumor agents. Early reports showed gramicidin inhibits human gastric cancer cell proliferation, cell cycle and induce apoptosis (Chen et al., 2019). As well as gramicidin blocks tumor growth and angiogenesis in renal cell carcinoma (David et al., 2014). When we analyzed the morphology of tumor cells pre-treated with Neo-Poly gramicidin, the plasmatic cell membrane was strongly affected after exposition with antibiotic solution. These results are supported on our knowledge about the mechanism of action of 


\section{Antitumor effect of Neomycin on human cervix cancer cells}

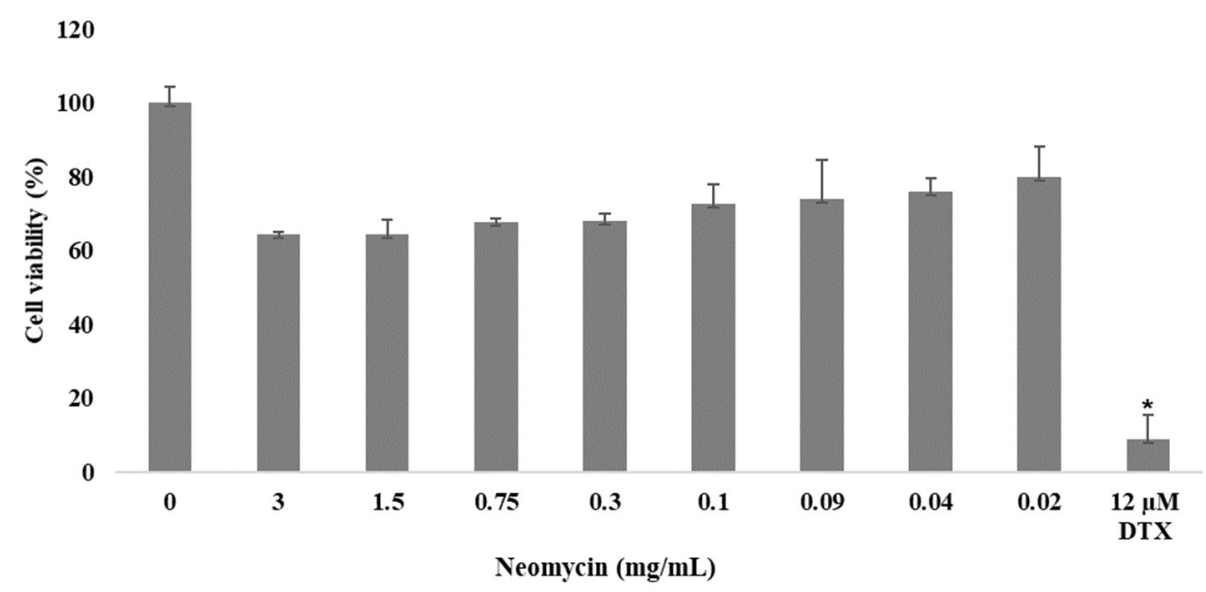

FIGURE 3 | Antitumor effect of neomycin on HeLa cells. Cell viability was evaluated with the MTT cell viability assay after a 24-h exposure to 0, 3, 1.5, 0.75, 0.3, 0.1, $0.09,0.04$, and 0.02 of neomycin and $12 \mu \mathrm{M}$ docetaxel (DTX) as positive control of cytotoxicity. Asterisks indicate statistical differences $(p<0.0001)(\alpha=0.05)$.



FIGURE 4 | Antitumor effect of polymyxin B on HeLa cell line. Cell viability (via MTT assay) after a 24-h exposure to serial dilutions of polymyxin B and $12 \mu \mathrm{M}$ docetaxel (DTX) as positive control of cytotoxicity. Antitumor activity was significant and dose-dependent as of $0.75 \mathrm{mg} / \mathrm{mL}(p<0.0001)$. Asterisks indicate statistical differences $(\alpha=0.05)$.

gramicidin and polymyxin B, which interfere with lipid bilayer of biomembranes (Oh et al., 2017; Azar et al., 2018). Next, we tried to get insights about which of the three antibiotic compounds (neomycin, polymixin B or gramicidin) was predominately responsible for the antitumor effect on HeLa cells. At the same final concentration $(0.1 \mathrm{mg} / \mathrm{mL})$, HeLa growth inhibition was strongest with gramicidin (50\%) as compared to neomycin and polymixin B, respectively 26 and $11 \%$ growth inhibition. Recently it was reported that $40 \mathrm{nM}$ of gramicidin was necessary to block proliferation of gastric tumor cells (Chen et al., 2019). The addition of $0.3-3 \mu \mathrm{M}$ of gramicidin induced apoptosis after $2 \mathrm{~h}$ exposotion among gastric tumor cells (Chen et al., 2019). Thus, we identified gramicidin to be etiological agent of the antitumor activity against HeLa cells.

Unfortunately monotherapy that involve the employ of a single drug is not enough to suppress the tumor growth in patients. Combination therapy is an alternative of treatment in conventional chemotherapy that uses more than one drug and has been suggested to obtain synergistic effect increasing treatment efficacy and decrease the duration of treatment (Gurunathan et al., 2018). Nanomaterials are being used in cancer therapy due their biocidal properties increasing therapeutic efficiency, and reducing non-desired effects improving target delivery in the site where effect is needed (Davis et al., 




FIGURE 5 | Antitumor effect of gramicidin on HeLa cell line. Cell viability (via MTT assay) after a 24-h exposure to serial dilutions of gramicidin $12 \mu \mathrm{M}$ docetaxel (DTX) as positive control of cytotoxicity. Antitumor effect was significant and dose-dependent as of $0.04 \mathrm{mg} / \mathrm{mL}$ gramicidin $(p<0.0001)$. Asterisks indicate statistical difference with $0.02 \mathrm{mg} / \mathrm{mL}$ gramicidin $(\alpha=0.05)$.



2008). When we combined gramicidin with BisBAL NPs, there was a synergistic effect on tumor cell growth inhibition, in comparison to their efficacy individually. It was described by Zhao et al. that C-C chemokine ligand two recruits bismuth selenide nanoparticles to facilities photothermal sensitivity of breast cancer cells (Zhao et al., 2018). Several examples of combination therapy implicating metal nanoparticles can be underline; cervical carcinoma-targeted delivery of doxorubicin with folic acid-modified selenium nanoparticles has been tested successfully in vitro and in vivo (Xia et al., 2018). Calcium phosphate nanostructures constitute right now an alternative for combined drug delivery to target cancer cells based on their biocompatibility and excellent chemical properties (Son and Kim, 2017). Gold nanoparticles plus doxorubicin showed improved therapeutic efficacy against breast cancer cells and few toxicity (Li et al., 2018).

Previously, we have reported that the mechanism of action of BisBAL NPs against tumor cells involved altering the cell membrane and damaging genomic DNA (Hernandez-Delgadillo et al., 2018; Cabral-Romero et al., 2020). However, we did 


\section{Effect of BisBAL NPs-Gramicidin on cell membrane integrity of HeLa cells}
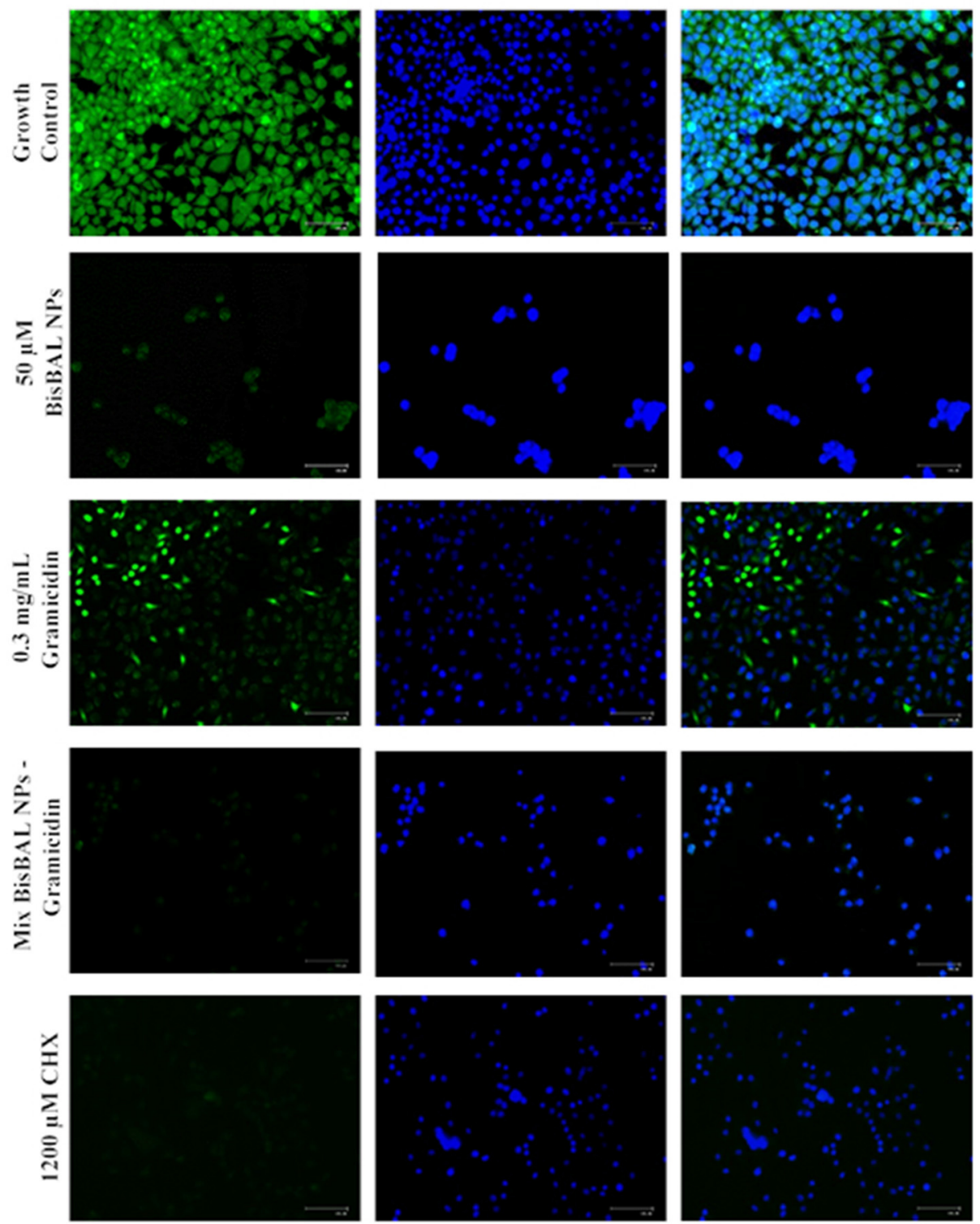

FIGURE 7 | Cell membrane permeability of HeLA cells was evaluated after treatment with gramicidin, BisBAL NPs or both by Calcein AM and fluorescence microscopy. A 24-h exposure to $0.3 \mathrm{mg} / \mathrm{mL}$ gramicidin, $50 \mu \mathrm{M}$ BisBAL NPs, or a BisBAL NPs/gramicidin mixture on HeLa cell monolayers was evaluated by calcein AM assay. Untreated cells were used as growth control and 1,200 $\mu \mathrm{M}$ chlorhexidine (CHX) served as a positive control. Bar indicates $125 \mu \mathrm{m}$.

not know the action mechanism of gramicidin on tumor cells. Gramicidin is a peptide of 15 amino acids and gramicidin dimers can form ion channel-like pores in cell membranes of bacteria (Gordon et al., 1943). Interestingly, doxorubicin interacts with the cell membrane of tumor cells causing physical alterations in the cell membrane in a dose-dependent way and promoting cell death. When cell membrane integrity was evaluated, HeLa cells $24 \mathrm{~h}$-exposed to $0.3 \mathrm{mg} / \mathrm{mL}$ of gramicidin, $50 \mu \mathrm{M}$ of BisBAL
NPs or a combination of both drugs, the results showed a strong damage on cell membrane and it was more evident after treatment with the mixture of gramicidin/BisBAL NP. These results support the hypothesis of synergistic phenomenon between gramicidin and BisBAL NPs. Also these data confirm previous reports about gramicidin's capacity to bind to cell membranes and alter their permeability. As expected, an effect on the nuclei of tumor cells was only observed for BisBAL 


\section{Genotoxic effect of BisBAL NPs-Gramicidin on HeLa cells}

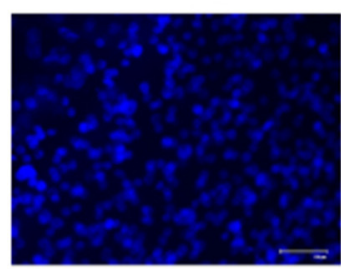

Growth

Control



$50 \mu \mathrm{M}$

BisBAL NPs

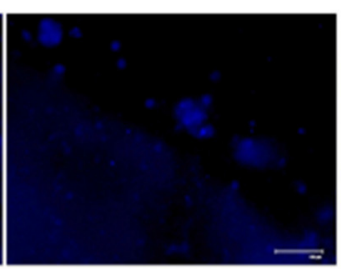

$0.3 \mathrm{mg} / \mathrm{mL}$

Gramicidin

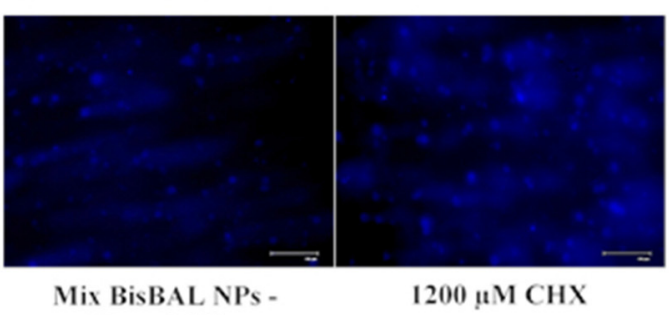

Gramicidin

FIGURE 8 | Genotoxic effect of gramicidin alone or in combination with BisBAL NPs on HeLa cells. Tumor cells were exposed for $24 \mathrm{~h}$ to $0.3 \mathrm{mg} / \mathrm{mL}$ gramicidin, $50 \mu \mathrm{M}$ of BisBAL NPs, or a BisBAL NPs/gramicidin mixture. 1,200 $\mu$ M chlorhexidine served as a positive control of genotoxic effect. DNA damage was analyzed by the comet assay and fluorescence microscopy. Bar indicates $125 \mu \mathrm{m}$.

NPs, which is consistent with previous reports (HernandezDelgadillo et al., 2018), but not for gramicidin. Thus, it seems that gramicidin only attacks the cell membrane of HeLa cells. Together, gramicidin and BisBAL NP seem highly synergistic: gramicidin alters membrane integrity and BisBAL NP is genotoxic.

In conclusion, we report in this study the effectiveness of the triple antibiotic solution Neo-Poly gramicidin as a growth inhibitor of the human cervical cancer cells known as HeLa. The combination of gramicidin/BisBAL NPs showed a synergistic antitumor activity on HeLa, probably due to a combination of altered cell membrane integrity of tumor cells and a genotoxic effect. The gramicidin/BisBAL NP combination may be a promising alternative for the topical treatment of cervical carcinoma.

\section{REFERENCES}

Adegoke, O., Kulasingam, S., and Virnig, B. (2012). Cervical cancer trends in the United States: a 35-year population-based analysis. J. Womens Health (Larchmt) 21, 1031-1037. doi: 10.1089/jwh.201 1.3385

Azar, E., Constantin, D., and Warschawski, D. E. (2018). The effect of gramicidin inclusions on the local order of membrane components. Eur. Phys. J. E. Soft Matter 41, 44. doi: 10.1140/epje/i2018-11644-5

Badireddy, A. R., Hernandez-Delgadillo, R., Sánchez-Nájera, R. I., Chellam, S., and Cabral-Romero, C. (2014). Synthesis and characterization of lipophilic bismuth dimercaptopropanol nanoparticles and their effects on oral microorganisms growth and biofilm formation. J. Nanopart. Res. 16:2456. doi: 10.1007/s11051-014-2456-5

Bosscha, M. I., Van Dissel, J. T., Kuijper, E. J., Swart, W., and Jager, M. J. (2004). The efficacy and safety of topical polymyxin B, neomycin and gramicidin for treatment of presumed bacterial corneal ulceration. Br. J. Ophthalmol. 88, 25-28. doi: 10.1136/bjo.88.1.25

Bozyczko-Coyne, D., Mckenna, B. W., Connors, T. J., and Neff, N. T. (1993). A rapid fluorometric assay to measure neuronal survival in vitro. J. Neurosci. Methods 50, 205-216. doi: 10.1016/0165-0270(93)90009-G

Cabral-Romero, C., Solis-Soto, J. M., Sanchez-Perez, Y., Pineda-Aguilar, N., Meester, I., Perez-Carrillo, E., et al. (2020). Antitumor activity of a hydrogel loaded with lipophilic bismuth nanoparticles on cervical, prostate, and colon human cancer cells. Anticancer Drugs 31, 251-259. doi: 10.1097/CAD.0000000000000863

\section{DATA AVAILABILITY STATEMENT}

The raw data supporting the conclusions of this article will be made available by the authors, without undue reservation.

\section{AUTHOR CONTRIBUTIONS}

All authors listed have made a substantial, direct and intellectual contribution to the work, and approved it for publication.

\section{FUNDING}

The authors thank the Sectorial Fund for Education Research, CONACyT, CB 2017-2018 for grant A1-S-20148.

Chen, T., Wang, Y., Yang, Y., Yu, K., Cao, X., Su, F., et al. (2019). Gramicidin inhibits human gastric cancer cell proliferation, cell cycle and induced apoptosis. Biol. Res. 52::57. doi: 10.1186/s40659-019-0264-1

Clarke, M. A., Wentzensen, N., Mirabello, L., Ghosh, A., Wacholder, S., Harari, A., et al. (2012). Human papillomavirus DNA methylation as a potential biomarker for cervical cancer. Cancer Epidemiol. Biomarkers Prev. 21, 2125-2137. doi: 10.1158/1055-9965.EPI-12-0905

David, J. M., Owens, T. A., Inge, L. J., Bremner, R. M., and Rajasekaran, A. K. (2014). Gramicidin A blocks tumor growth and angiogenesis through inhibition of hypoxia-inducible factor in renal cell carcinoma. Mol. Cancer Ther. 13, 788-799. doi: 10.1158/1535-7163.MCT-13-0891

Davis, M. E., Chen, Z. G., and Shin, D. M. (2008). Nanoparticle therapeutics: an emerging treatment modality for cancer. Nat. Rev. Drug Discov. 7, 771-782. doi: $10.1038 / \mathrm{nrd} 2614$

Elit, L., Fyles, A. W., Devries, M. C., Oliver, T. K., and Fung-Kee-Fung, M. (2009). Follow-up for women after treatment for cervical cancer: a systematic review. Gynecol. Oncol. 114, 528-535. doi: 10.1016/j.ygyno.2009.06.001

Ferlay, J., Steliarova-Foucher, E., Lortet-Tieulent, J., Rosso, S., Coebergh, J. W., Comber, H., et al. (2013). Cancer incidence and mortality patterns in Europe: estimates for 40 countries in 2012. Eur. J. Cancer 49, 1374-1403. doi: 10.1016/j.ejca.2012.12.027

Gibson, J. R. (1983). Trimethoprim-polymyxin B ophthalmic solution in the treatment of presumptive bacterial conjunctivitis-a multicentre trial of its efficacy versus neomycin-polymyxin B-gramicidin and chloramphenicol ophthalmic solutions. J. Antimicrob. Chemother. 11, 217-221. doi: 10.1093/jac/11.3.217 
Gordon, A. H., Martin, A. J., and Synge, R. L. (1943). The amino-acid composition of gramicidin. Biochem. J. 37, 86-92. doi: 10.1042/bj0370086

Gurunathan, S., Kang, M.-H., Qasim, M., and Kim, J.-H. (2018). Nanoparticlemediated combination therapy: two-in-one approach for cancer. Int. J. Mol. Sci. 19:3264. doi: 10.3390/ijms19103264

Hernandez-Delgadillo, R., Garcia-Cuellar, C. M., Sanchez-Perez, Y., PinedaAguilar, N., Martinez-Martinez, M. A., Rangel-Padilla, E. E., et al. (2018). In vitro evaluation of the antitumor effect of bismuth lipophilic nanoparticles (BisBAL NPs) on breast cancer cells. Int. J. Nanomed. 13, 6089-6097. doi: 10.2147/IJN.S179095

Hu, G. F. (2001). Neomycin inhibits the angiogenic activity of fibroblast and epidermal growth factors. Biochem. Biophys. Res. Commun. 287, 870-874. doi: 10.1006/bbrc.2001.5668

Kitaura, K., Watanabe, Y., Ishihara, Y., and Takahira, H. (1972). [Antitumor activity of adriamycin]. Jpn. J. Antibiot. 25, 65-71. doi: 10.7164/antibiotics.25.509

Kubista, M., Akerman, B., and Norden, B. (1987). Characterization of interaction between DNA and 4,6-diamidino-2-phenylindole by optical spectroscopy. Biochemistry 26, 4545-4553. doi: 10.1021/bi00388a057

Li, Y., He, D., Tu, J., Wang, R., Zu, C., Chen, Y., et al. (2018). The comparative effect of wrapping solid gold nanoparticles and hollow gold nanoparticles with doxorubicin-loaded thermosensitive liposomes for cancer thermo-chemotherapy. Nanoscale 10, 8628-8641. doi: 10.1039/C7NR $09083 \mathrm{H}$

Liu, Y., Peterson, D. A., Kimura, H., and Schubert, D. (1997). Mechanism of cellular 3-(4,5-dimethylthiazol-2-yl)-2,5-diphenyltetrazolium bromide (MTT) reduction. J. Neurochem. 69, 581-593. doi: 10.1046/j.1471-4159.1997.69020581.x

Martin-Hirsch, P. P., Paraskevaidis, E., Bryant, A., and Dickinson, H. O. (2013). Surgery for cervical intraepithelial neoplasia. Cochrane Database Syst. Rev. CD001318. doi: 10.1002/14651858.CD001318.pub3

Nascimento, A. V., Singh, A., Bousbaa, H., Ferreira, D., Sarmento, B., and Amiji, M. M. (2017). Overcoming cisplatin resistance in nonsmall cell lung cancer with Mad2 silencing siRNA delivered systemically using EGFR-targeted chitosan nanoparticles. Acta Biomater. 47, 71-80. doi: 10.1016/j.actbio.2016.09.045

Oh, Y. J., Plochberger, B., Rechberger, M., and Hinterdorfer, P. (2017). Characterizing the effect of polymyxin B antibiotics to lipopolysaccharide on Escherichia coli surface using atomic force microscopy. J. Mol. Recognit. 30:2605. doi: $10.1002 / \mathrm{jmr} .2605$

Ostling, O., and Johanson, K. J. (1984). Microelectrophoretic study of radiationinduced DNA damages in individual mammalian cells. Biochem. Biophys. Res. Commun. 123, 291-298. doi: 10.1016/0006-291X(84)90411-X

Rene, H.-D., Badireddy, A. R., Juan José, M.-S., Juan Francisco, C.-C., Gustavo Israel, M.-G., Rosa Isela, S.-N., et al. (2016). Cytotoxic effect of lipophilic bismuth dimercaptopropanol nanoparticles on epithelial cells. J. Nanosci. Nanotechnol. 16, 203-209. doi: 10.1166/jnn.2016.10735

Rivankar, S. (2014). An overview of doxorubicin formulations in cancer therapy. $J$. Cancer Res. Ther. 10, 853-858. doi: 10.4103/0973-1482.139267

Seol, H. J., Ulak, R., Ki, K. D., and Lee, J. M. (2014). Cytotoxic and targeted systemic therapy in advanced andrecurrent cervical cancer: experience from clinical trials. Tohoku J. Exp. Med. 232, 269-276. doi: 10.1620/tjem.2 32.269

Serrano-Olvera, A., Cetina, L., Coronel, J., and Duenas-Gonzalez, A. (2015). Emerging drugs for the treatment of cervical cancer. Expert. Opin. Emerg. Drugs 20, 165-182. doi: 10.1517/14728214.2015.1002768

Shi, J., Kantoff, P. W., Wooster, R., and Farokhzad, O. C. (2017). Cancer nanomedicine: progress, challenges and opportunities. Nat. Rev. Cancer 17, 20-37. doi: $10.1038 /$ nrc.2016.108

Son, K. D., and Kim, Y. J. (2017). Anticancer activity of drug-loaded calcium phosphate nanocomposites against human osteosarcoma. Biomater. Res. 21:13. doi: 10.1186/s40824-017-0099-1

Torre, L. A., Bray, F., Siegel, R. L., Ferlay, J., Lortet-Tieulent, J., and Jemal, A. (2015). Global cancer statistics, 2012. CA Cancer. J. Clin. 65, 87-108. doi: $10.3322 /$ caac. 21262

Villa, A., Patton, L. L., Giuliano, A. R., Estrich, C. G., Pahlke, S. C., O'brien, K. K., et al. (2020). Summary of the evidence on the safety, efficacy, and effectiveness of human papillomavirus vaccines: Umbrella review of systematic reviews. J. Am. Dent. Assoc. doi: 10.1016/j.adaj.2019.10.010

Wang, Z., Liang, X., Yu, J., Zheng, X., Zhu, Y., Yan, Y., et al. (2012). Nongenetic risk factors and predicting efficacy for docetaxel-drug-induced liver injury among metastatic breast cancer patients. J. Gastroenterol. Hepatol. 27, 1348-1352. doi: 10.1111/j.1440-1746.2012.07131.x

Waterhouse, D. N., Tardi, P. G., Mayer, L. D., and Bally, M. B. (2001). A comparison of liposomal formulations of doxorubicin with drug administered in free form: changing toxicity profiles. Drug Saf. 24, 903-920. doi: 10.2165/00002018-200124120-00004

Xia, Y., Xu, T., Zhao, M., Hua, L., Chen, Y., Wang, C., et al. (2018). Delivery of doxorubicin for human cervical carcinoma targeting therapy by folic acid-modified selenium nanoparticles. Int. J. Mol. Sci. 19:19113582. doi: $10.3390 /$ ijms 19113582

Yared, J. A., and Tkaczuk, K. H. (2012). Update on taxane development: new analogs and new formulations. Drug Des. Devel. Ther. 6, 371-384. doi: 10.2147/DDDT.S28997

Zhao, H., Li, L., Zhang, J., Zheng, C., Ding, K., Xiao, H., et al. (2018). C-C Chemokine Ligand 2 (CCL2) recruits macrophage-membrane-camouflaged hollow bismuth selenide nanoparticles to facilitate photothermal sensitivity and inhibit lung metastasis of breast cancer. ACS Appl. Mater. Interfaces 10, 31124-31135. doi: 10.1021/acsami.8b11645

Conflict of Interest: The authors declare that the research was conducted in the absence of any commercial or financial relationships that could be construed as a potential conflict of interest.

Copyright () 2021 Cabral-Romero, García-Cuellar, Hernandez-Delgadillo, SánchezPérez, Meester, Solis-Soto, Pineda-Aguilar and Chellam. This is an open-access article distributed under the terms of the Creative Commons Attribution License (CC $B Y)$. The use, distribution or reproduction in other forums is permitted, provided the original author(s) and the copyright owner(s) are credited and that the original publication in this journal is cited, in accordance with accepted academic practice. No use, distribution or reproduction is permitted which does not comply with these terms. 